\title{
A Robot Assisted Hip Fracture Reduction with a Navigation System ${ }^{\star}$
}

\author{
Sanghyun Joung ${ }^{1}$, Hirokazu Kamon ${ }^{2}$, Hongen Liao ${ }^{2}$, Junichiro Iwaki ${ }^{6}$, \\ Touji Nakazawa ${ }^{6}$, Mamoru Mitsuishi², Yoshikazu Nakajima ${ }^{2}$, \\ Tsuyoshi Koyama ${ }^{4}$, Nobuhiko Sugano ${ }^{4}$, Yuki Maeda ${ }^{5}$, Masahiko Bessho $^{3}$, \\ Satoru Ohashi ${ }^{3}$, Takuya Matsumoto ${ }^{3}$, Isao Ohnishi ${ }^{3}$, and Ichiro Sakuma ${ }^{2}$ \\ ${ }^{1}$ Graduate School of Frontier Sciences, the University of Tokyo, Japan \\ shjoung@bmpe.t.u-tokyo.ac.jp \\ ${ }^{2}$ Graduate School of Engineering, the University of Tokyo, Japan \\ ${ }^{3}$ Graduate School of Medicine, the University of Tokyo, Japan \\ ${ }^{4}$ Graduate School of Medicine, Osaka University, Japan \\ ${ }^{5}$ National Organization Osaka Minami Medical Center, Japan \\ ${ }^{6}$ THK CO.,LTD. Japan
}

\begin{abstract}
A fracture reduction robot is described as assisting in safe and precise fracture reduction. The robot is connected with pins that are inserted into the patient's bone fragments, together with a customized jig. The robot has six degrees of freedom with high precision, so that precise fracture reduction can be conducted. The failsafe unit of the fracture reduction robot can mitigate excessive reduction force that may cause complications such as avascular necrosis. We have integrated the fracture reduction robot with a navigation system that tracks the relative position of the bone fragments and generates the reduction path. The integrated system is evaluated with the simulated fracture reduction of a hip fracture model $(n=8)$. Three-dimensional parameters related to the mechanical axis-the proximal femur angle, the distal femur angle, and the length of the mechanical axis-were evaluated by comparing the normal values with those after reduction; these average differences are $1.76^{\circ}, 0.28^{\circ}$ and $0.76 \mathrm{~mm}$, respectively. The automated fracture reduction feature makes it possible for medical staff to work at a distance from radiation sources; for patients, the integrated fracture reduction system has the potential to reduce fractures with high precision.
\end{abstract}

\section{Introduction}

Femoral neck fractures can occur in the elderly, on account of simple falls; incidences thereof are increasing in our aging society. Most patients are bedridden and should be surgically treated. Though surgery outcomes are influenced by a fracture reduction accuracy, highly qualified medical care and repeated radiation exposure are both required.

* We wish to thank many people and groups who have contributed to this effort. Research on medical devices for analyzing, supporting and substituting the function of human body funded by Ministry of Health, Labour and Welfare(H17-Physi-007). 
Surgical navigation has been previously applied to guidance in fracture reduction [1. Computer-assisted surgery can help reduce reliance on fluoroscopy and improve the accuracy of reduction. Robot-assisted fracture reduction is a good solution for these problems, and several groups have reported the development of this sort of robot [2] 3. While those robots are typically modifications of industrial robots, we have developed a fracture reduction robot designed expressly for medical use [4].

Our robot has six DOF and a mechanical failsafe mechanism; it also has a power-assisted control mode and an automatic operation mode in the navigation system. However, there were some issues with this robot that must be rectified. The robot uses a traction boot for fracture reduction, in much the same way the conventional manual reduction method uses a fracture table. It is, for that reason, difficult to position the bone fragments precisely, in spite of the increased DOF. Even when connectivity between the reduction robot and the surgical navigation has been confirmed, it is necessary to evaluate the clinical usefulness of the integrated system that involves navigation.

In this paper, we present a fracture reduction assisting system that consists of a fracture reduction robot and a navigation system. For the accurate positioning of bone fragments, bone fragments are directly connected with the robot using a customized jig. The navigation system tracks the positions of bone fragments and generates an appropriate reduction path. The clinical usefulness of the system was evaluated using experimental results from a simulated fracture reduction.

\section{Method}

We carefully considered safety, mobility, precision of control, and ease of extension to other machines-such as navigation systems-in developing the fracture reduction robot. In this section, which focuses on the target operation, the apparatus of the fracture reduction robot, the navigation system, and the integration of the two are described.

\subsection{Target Operation}

Conventional fracture reduction with the use of a fracture table is an indirect reduction method. This method is not invasive, but it is difficult for surgeons to reduce precisely, due to force absorption at soft tissues such as joints, skins, muscles, and the like. For more precise positioning of bone fragments, a direct reduction method-such as the one proposed by our medical team-is required for the target operative technique. In executing that technique, two medical screws are inserted into the bone fragment and connected to the ring frame, so that the connection between the ring frame and the bone fragments is very rigid. The bone fragment can be arranged precisely by handling the ring frame, though this method does require a small incision. 


\subsection{Fracture Reduction Robot}

An outline of the fracture reduction robot is provided in Figure 1(a); a kinematic model is provided in Figure1(c). The robot has six independent DOFs (i.e., three DOFs in translation and three DOFs in rotation). Three rotational axes intersect each other at one point, for easy control from the kinematics calculation point of view. Four steerable wheels attached beneath the robot enable medical staff to move the robot easily, in spite of its weight $(315 \mathrm{~kg})$. The size of the robot is $640 \times 1084 \times 1317 \mathrm{~mm}($ width $\times$ long $\times$ height $)$. It can be transported with the use of a common passenger elevator.

The customized jig, shown in Figure 1(b), connects the bone fragment with the robot. Carbon fiber and duralumin were used as the main materials of the jig, making it lightweight yet highly rigid. The position and posture of the jig can be adjusted with six DOFs, depending on the relative position between the bone fragment and the fracture reduction robot. The setup and connection of the robot to the patient is relatively simple and straightforward.

For safety assurance, two mechanical failsafe units, a six-axis force sensor, and emergency stop buttons are installed in the system. The mechanical failsafe units can mitigate excessive reduction force, and the units maintain high rigidity within the allowed force and torque ranges. However, if an excessive force is applied to the unit, it decouples the customized jig from the actuation unit to remove the applied force or torque, so that the bone fragment is prevented from any excessive movement that could damage the precarious blood supply. One of the two mechanical failsafe units is used to limit the application force along
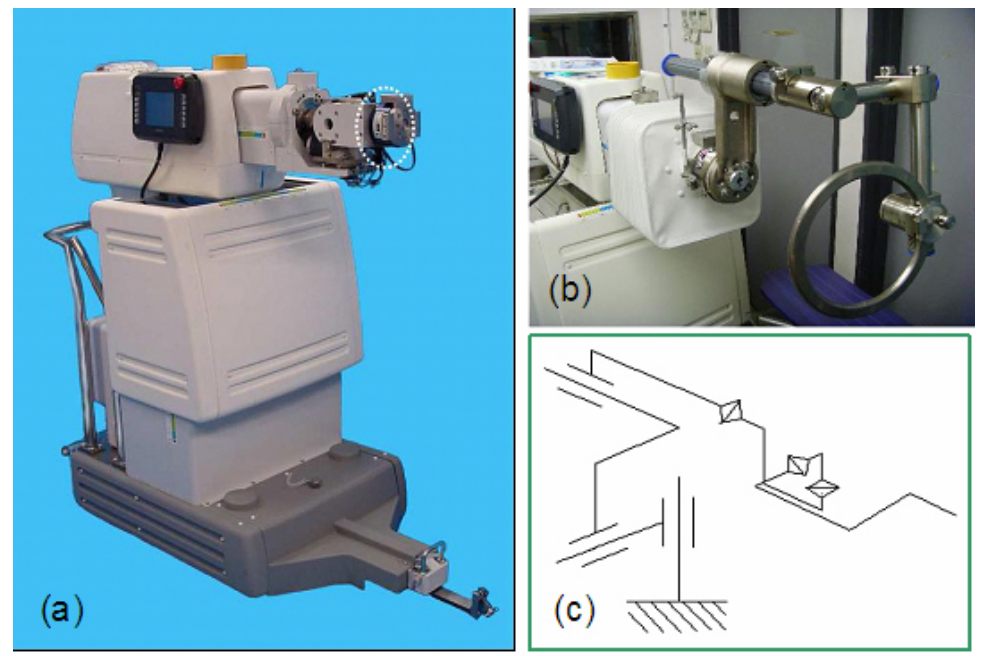

Fig. 1. The fracture reduction robot. (a) the outline of the robot. a six-axis force sensor is shown in the white dotted circle, (b) the customized jig for connecting the bone fragment with the robot, and (c) the kinematic model of the robot. 
the traction direction, and the other unit is used to limit the rotation torque around the internal or external rotation axis. The thresholds force and torque is designed to refer the clinical data reported by Maeda et al [5], and these can be adjusted from $200 N$ to $400 N$, and from $20 N m$ to $30 N m$ with accuracy of $10 \%$, respectively. The reduction force is also monitored by the six-axis force sensor, which is mounted on the robot as shown in the white dotted circle of Figure 1(a). The measured reduction force is used to manage the software failsafe system, to keep the applied force and torque within the allowed ranges. When a surgeon or medical staff pushes the emergency stop button, each axis of the robot stops its operation and the actuation units are decoupled, so that the customized jig can be operated manually.

\subsection{Navigation System}

The navigation system has two main functions. One is to track the relative position between the bone fragments and the robot; the other is to generate an appropriate reduction path. In this system, we plan to use 2D-3D registration of the fluoroscopic image to the three-dimensional preoperative computed tomography (CT) data. An optical 3D position measurement system (Optotrack, NDI, Canada) is used to track the intraoperative fluoroscope system, the reduction robot's position, and the bone fragments.

The tracking method combines $2 \mathrm{D}$ and $3 \mathrm{D}$ image registration from single-view fluoroscopy with tracking of the head center position of the proximal femoral fragment, to improve the accuracy of fluoroscopic registration-all without the need for the repeated manual adjustment of the $\mathrm{C}$-arm that is required with stereo-view registrations. Kinematic knowledge of the hip joint, which has a positional correspondence with the femoral head center and the pelvis acetabular center, allows the position of the femoral fragment to be determined from pelvis tracking [6]. The robot's reference marker is used not only for tracking the robot, but also for tracking the distal bone fragment. The position of the distal bone can be calculated using the robot's position, since the distal bone is rigidly connected to the robot. The reduction path is generated using the acquired relation of the bone fragments, and it is visualized through the use of animation. The generated reduction path is interactively edited by the surgeon, and the generated reduction path is transmitted to the robot.

\subsection{System Configuration and Surgical Procedure}

The system configuration is illustrated in Figure 2. The optical 3D position measurement system tracks the proximal and distal bones, and it transmits the measured data to the navigation system. The navigation system tracks the relative spacing between the bone fragments and the robot. The intraoperative fluoroscopy system, the surgical navigation system, and the robot controller communicate with each other through the computer network (TCP/IP); the navigation system sends control commands to the robot, and the robot replies with the current status. 
The control process of the robot is managed in real time, with a frequency of 1 $\mathrm{KHz}$. So as not to interfere with the control process during data communication, commands from the navigation system are handled by a user task that is a nonreal-time process. The command data is written in the shared memory on a first in, first out (FIFO) basis, and they are processed by the FIFO handler of the control routine.

The surgical procedure by the integrated system for fracture reduction is as follows:

Step 1) The three-dimensional surface data of the bone fragments are estimated from CT scans of the injured hip, prior to surgery.

Step 2) A reference sensor is attached to the pelvis.

Step 3) The coordinates of the fracture reduction robot are calibrated by the navigation system.

Step 4) The distal bone fragment is connected to the robot, using the customized jig.

Step 5) The coordinates of the bone fragments are calculated with 2D-3D registration, following fluoroscopic scan.

Step 6) The navigation system tracks the relative position of the bone fragments until the reduction is complete.

Step 7) The navigation system generates the reduction path and the surgeon interactively corrects it, as needed.

Step 8) The robot reduces the fracture, following the commands from the navigation system.

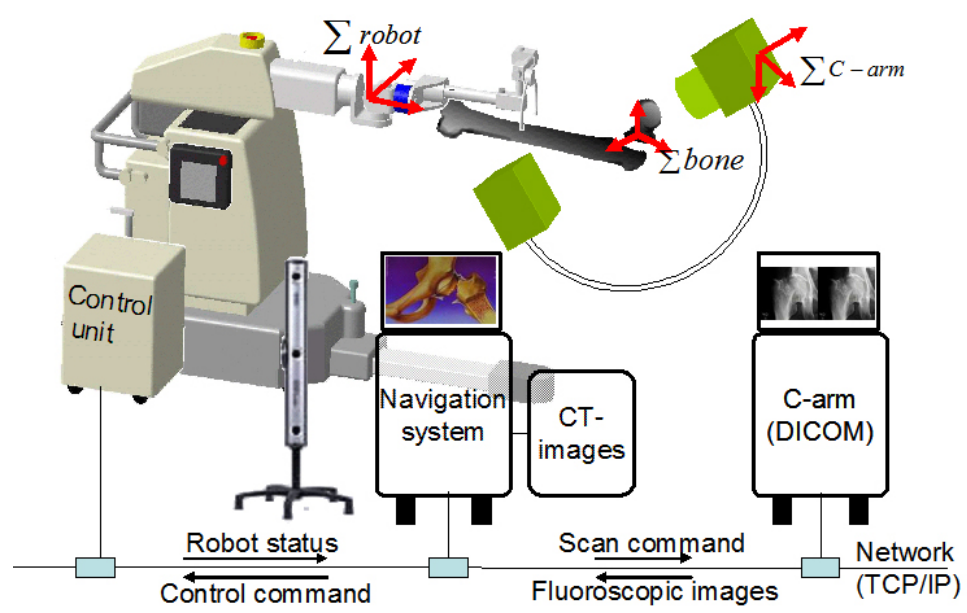

Fig. 2. Configuration of the integrated system, between the fracture reduction robot and the navigation system 


\section{Results}

\subsection{Simulated Fracture Reduction}

We prepared a femoral neck-fracture model by cutting the femur model (SAWBONES, Pacific Research Laboratories, Inc. USA) with a band saw and attaching a rubber band between the femur and the hip, to simulate the influence of muscles such as the gluteus medius and the piriformis. The fracture reduction was conducted following the procedure described in section 2.4. with the exception of step 5. A pen-type reference marker was used to trace the surface of the bone fragments instead of fluoroscope, and the acquired data were combined with the surface data acquired in step 1 using the surface matching method.

We corrected the reduction path referred to in the common reduction method, which pulls down the distal bone a little and locates the correct position with internal rotation. It takes about one minute to reduce the fracture with the robot (step 8); the "before" and "after" of the reduction are shown in Figure 3 .
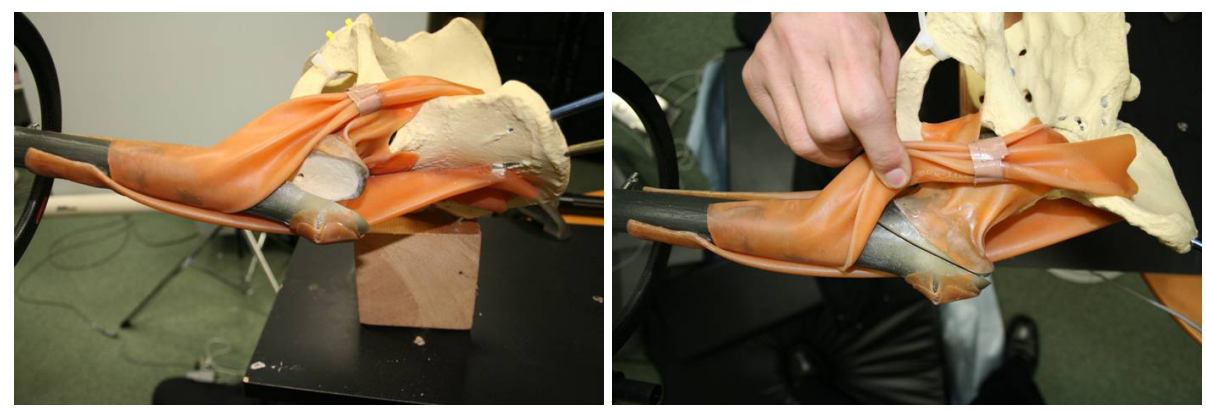

Fig. 3. Results of fracture reduction, (a) before reduction and (b) after reduction

\subsection{Evaluation Method and Experimental Results}

A fracture reduction is generally assessed using two-dimensional fluoroscopic images. The reduction alignment angles of the anteroposterior (AP) and lateral views should be within the defined values; however, this assessment is influenced by the measured angle of the fluoroscopic images, as well as the surgeon's viewpoint. Therefore, to ensure high reproducibility, we evaluated the reduction result from parameters related to the mechanical axis used to assess the femur deformity. The mechanical axis is drawn from the center of the knee joint to the head of the femur, as illustrated in Figure4. If the mechanical axis is defined, the mechanical distal femur angle (DFA) and proximal femur angle (PFA) can also be defined. Before fracturing the femur model, we marked feature points such as the greater trochanter, the head of the femur, the lateral condyle, and the medical condyle; these points are shown as red points in Figure 4. We measured the three-dimensional positions of the feature points using the pen-type reference marker and the optical 3D position measurement system; we then calculated the 


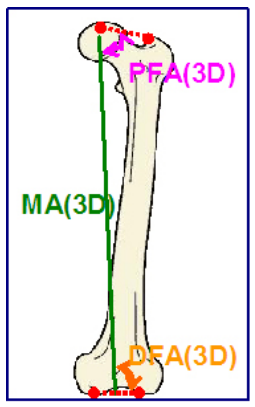

\begin{tabular}{|l|r|r|r|r|r|r|r|}
\hline \multirow{2}{*}{} & \multirow{2}{*}{ Normal } & \multicolumn{3}{|c|}{ Reduction value } & \multicolumn{3}{|c|}{ Difference } \\
\cline { 3 - 8 } & & ave & $\min$ & $\max$ & ave & $\min$ & max \\
\hline $\begin{array}{l}\text { PFA } \\
\text { (degree) }\end{array}$ & 88.14 & 89.90 & 89.08 & 90.59 & 1.76 & 0.94 & 2.45 \\
\hline $\begin{array}{l}\text { DFA } \\
\text { (degree) }\end{array}$ & 90.60 & 90.80 & 90.26 & 91.36 & 0.28 & 0.05 & 0.71 \\
\hline MA (mm) & 426.78 & 427.86 & 425.49 & 428.86 & 0.76 & 0.06 & 2.09 \\
\hline
\end{tabular}

Fig. 4. Femoral mechanical axis, and the related parameters and results $(n=8)$

"normal values" of the length of the mechanical axis, the PFA, and the DFA from the four measured feature points. The "reduction values" following fracture reduction were finally compared to the normal values. The table in Figure 4 shows the normal values, reduction values, and differences thereof.

\section{Discussion}

A fracture reduction procedure is generally considered accurate and precise when the alignment error and the gap are within $2^{\circ}$ and $2 \mathrm{~mm}$, respectively, according to assessments based on two-dimensional imagery. We compared threedimensional parameters, two angles, and a length, to assess the outcome of the simulated fracture reduction described in section 3.2. The average differences in PFA, DFA, and MA were $1.76^{\circ}, 0.28^{\circ}$ and $0.76 \mathrm{~mm}$, respectively $(\mathrm{n}=8)$. Although we offer no recommended values for the evaluation method used in this paper, it is believed that differences are allowable when comparing the two-dimensional recommend values, and that the integrated system has the potential to reduce fractures with high precision. The differences are interpreted as a combination of the registration error of the bone fragments, the robot motion artifact, and optical-tracking error.

The fracture model used in the experiment uses imitation muscles, to reproduce a fracture type similar to clinical status. The surgeons in our research group evaluated the fracture model comparable to the clinical cases. To evaluate the failsafe system, we must improve the fracture model. There are limitations in evaluating the system and the mechanical failsafe system in particular. The safety mechanism should be evaluated more clearly with the created fracture model, based on the clinical data; we are preparing to measure clinical datasuch as reduction force and the reduction path-during the direct fracture reduction. In our experiments, we could not use a fluoroscope for 2D-3D registration of bone fragments. We are trying to integrate our system and the registration method, which is reported by Nakajima et al [6]. Though the radiation exposure, the registration error, and the reduction accuracy of a complete system should be evaluated, it can be expected to reduce the radiation exposure, because the 
system shortens the overall reduction time and the surgeon can monitor the reduction status via the navigation system, without the use of a fluoroscope, and moreover surgeons can work at a distance from radiation source.

In conclusion, we have developed the fracture reduction robot to assist in fraction reduction, with high levels of precision and safety. The robot is connected to the bone fragment with pins that were inserted into the patient bone fragment with the customized jig. The robot has six degrees of freedom with high precision, so a precise fracture reduction can be performed by using the robot. The fracture reduction robot was integrated with a surgical navigation system that can track the relative positions of bone fragments, so the robot can generate an appropriate reduction path. The entire system was evaluated using simulated fracture reduction, and the results thereof show the ability of the proposed system in reducing fractures with high precision and thus shortening radiation exposure time. Nonetheless, the safety mechanism of the fracture reduction robot should be evaluated and the stability of the integrated system should also be evaluated.

\section{References}

1. Chong, K.W., Wong, M.K., Rikhraj, I.S., Howe, T.S.: The use of computer navigation in performing minimally invasive surgery for intertrochanteric hip fractures-the experience in singapore. Injury 37, 755-762 (2006)

2. Füchtmeier, B., Egersdoerfer, S., Mai, R., Hente, R., Dragoi, D., Monkman, G., Nerlich, M.: Reduction of femoral shaft fractures in vitro by a new developed reduction robot system 'reporobo'. Injury 35(1), 113-119 (2004)

3. Westphal, R., Gösling, T., Oszwald, M., Bredow, J., Klepzig, D., Winkelbach, S., Hüfner, T., Krettek, C., Wahl, F.: 3d robot assisted fracture reduction. In: Proceedings of 10th International Symposium on Experimental Robotics 2006 (ISER 2006), pp. 6-10 (2006)

4. Mitsuishi, M., Sugita, N., Warisawa, S., Ishizuka, T., Nakazawa, T., Sugano, N., Yenenobu, K., Sakuma, I.: Development of a computer-integrated femoral head fracture reduction system. In: Proceedings of ICM 2005, pp. 834-839 (2005)

5. Maeda, Y., Tamura, Y., Saito, M., Yamamoto, T., Yonenobu, K., Warisawa, S., Mitsuishi, M., Sugano, N.: Measurement of traction load and torque transferred to the lower extremity during simulated fracture reduction. International Congress Series 1281, 674-679 (2005)

6. Nakajima, Y., Tashiro, T., Sugano, N., Yonenobu, K., Koyama, T., Maeda, Y., Tamura, Y., Saito, M., Tamura, S., Mitsuishi, M., Sugita, N., Sakuma, I., Ochi, T., Matsumoto, Y.: Fluoroscopic bone fragment tracking for surgical navigation in femur fracture reduction by incorporating optical tracking of hip joint rotation center. IEEE Transactions on Biomedical Engineering 54(9), 1703-1706 (2007) 